\title{
Bioanalysis
}

\section{Boston Society's 11th Annual Applied Pharmaceutical Analysis conference}

\section{Boston Society's 11th Annual Applied Pharmaceutical Analysis conference, Hyatt Regency Hotel, Cambridge, MA, USA, 14-16 September 2015.}

The Boston Society's 11th Annual Applied Pharmaceutical Analysis (APA) conference took place at the Hyatt Regency hotel in Cambridge, MA, on 14-16 September 2015. The 3-day conference affords pharmaceutical professionals, academic researchers and industry regulators the opportunity to collectively participate in meaningful and relevant discussions impacting the areas of pharmaceutical drug development. The APA conference was organized in three workshops encompassing the disciplines of regulated bioanalysis, discovery bioanalysis (encompassing new and emerging technologies) and biotransformation. The conference included a short course titled 'Bioanalytical considerations for the clinical development of antibody-drug conjugates (ADCs)', an engaging poster session, several panel and round table discussions and over 50 diverse talks from leading industry and academic scientists.

First draft submitted: 24 November 2015; Accepted for publication: 25 November 2015; Published online: 8 February 2016

Keywords: APA • applied pharmaceutical analysis $\bullet$ bioanalysis $\bullet$ the Boston Society - conference report

The 2015 APA conference was a tremendous success and continues to provide a unique forum for attendees to network and freely share their science, ideas and experience. Summarized below are some highlights and key discussions of the 2015 APA conference.

\section{Regulated bioanalysis}

After an introduction to the conference by Dr Chandra Prakash (Agios Pharmaceuticals, Cambridge, MA), the plenary speaker for Day 1, Dr Michael Skelly (US FDA-CDER-OTSOSIS-DGDBE, Silver Spring, MD) [1] was introduced who spoke on the current state of the US FDA. Dr Skelly's talk was focused on changes to the statutes, regulations and guidance, as well as updates on the FDA's bioequivalence inspection program and how they are aligning resources to become more efficient.
Dr Skelly began his talk by stating that statutes are binding to organizations while guidance documents are suggestions. Draft guidance should be used as points of discussion with the FDA. Dr Skelly reviewed an amendment of the Biologics Price Competition and Innovation Act of 2009, part of the Patient Protection and Affordable Care Act of 2010. The Public Health Service Act (PHSA) was amended to include Section 351(k) 'licensure of biological products as biosimilar or interchangeable'. The language included partly follows EMEA guidelines. Dr Skelly also mentioned that most reference products for EMA 'biosimilars' and Canadian 'follow-on biologics' were approved in the USA as a New Drug Application (NDA). He also mentioned that most original Biologics License Application (BLA) remain under
Violet Lee', Ang Liu ${ }^{2}$ Elizabeth Groeber ${ }^{3}$, Mehran Moghaddam ${ }^{4}$, James Schiller ${ }^{5}$, Joseph A Tweed ${ }^{*, 6}$ \& Gregory S Walker ${ }^{6}$

${ }^{1}$ Genentech, Inc., South San Francisco, CA, USA

${ }^{2}$ Bristol-Myers Squibb, Analytical \& Bioanalytical Development, Princeton, NJ, USA

${ }^{3}$ WIL Research, Ashland, OH, USA ${ }^{4}$ Celgene Corp., Discovery DMPK, San Diego, CA, USA

${ }^{5}$ Merck \& Co., Regulated Bioanalytical - PPDM, West Point, PA, USA

${ }^{6}$ Pfizer, Inc., Worldwide Pharmacokinetics \& Metabolism - NCE, Groton, CT, USA

*Author for correspondence: Fax: 860.686.0775 joseph.tweed@pfizer.com 
patent protection and that the FDA has received several 351(k) applications. Dr Skelly then reviewed the key requirements for a biosimilar application as listed in PHSA 351 (k), Section (2)(A)(i)-Subsection (I->cc and IV) [2].

Dr Skelly then focused on the FDA Safety and Innovation Act of 2012 (FDASIA) and the Generic Drug User Fee Amendments of 2012 (GDUFA). He stated that this act authorized the collection of user fees to support the review of NDAs, BLAs, Abbreviated New Drug Application (ANDA) and biosimilars. These fees also support the continuing inspections of NDA and ANDA-related studies as well as bioequivalence sites. Dr Skelly detailed a new Inspection Efficiency Enhancements Program that has been developed and is supported by these fees. Highlights of this process include a risk-adjusted biennial surveillance inspection model for CGMP facilities. In addition, facility inspections will be prioritized by ANDA approvals lacking only an inspection. Dr Skelly also mentioned that a 5-year study of foreign regulators' inspections of CGMP facilities and bioequivalence sites will be developed to utilize foreign classifications as appropriate. A database of CGMP facilities and bioequivalence sites are available to industry on the FDA website [3]. Dr Skelly also discussed a partial separation of OSIS roles for bioequivalence and GLP inspections and an increased emphasis on a surveillance auditing approach. Dr Skelly closed by encouraging members of the industry to keep the lines of communication open with the FDA and to continue to practice good science.

Following the plenary session there was a regulated bioanalysis session focused on guidance in the industry and was chaired by Dr Johanna Mora (Bristol-Myers Squibb, Princeton, NJ) and Dr Eric Woolf (Merck \& Co, West Point, PA). The next speaker was Dr Kara Scheibner (US FDA-CDER-OTS-OSIS-DGDBE, Silver Spring, MD) [1] who discussed her experiences over the past year as an Office of Study Integrity and Surveillance (OSIS) investigator in the world of biologics. The objective of Dr Scheibner's talk was to discuss specific scientific issues that she observed and the discrepancies of experimental approaches encountered between firms during review of immunogenicity data. Dr Scheibner highlighted some key points to consider for your biologic assay methodology including, scientifically sound rationale, relevancy of controls to study samples and designing an assay to measure what is intended. Dr Scheibner's presentation focused on examples of antidrug antibody assays and stressed the need for careful selection of the positive control antibody. She also commented that the positive control should be as relevant as possible to the actual study samples and should be included as plate acceptance samples. Dr Scheibner then discussed nonreproducibility of results she has observed between Tier 1 and Tier 2 assays. She encouraged laboratories to have a consensus on how these data should be reported and whether nonreproducible results should trigger reanalysis or an investigation. Dr Scheibner also talked about assessing potential sources of nonspecific inhibition and optimizing the method of confirmatory cut point calculation accordingly. Lastly, Dr Scheibner discussed interferences in cell based neutralizing antibody assays. These assays add multiple layers of complexity so it is important to determine what type of cell is best suited. She encouraged scientist to assess all potential endogenous interferences in method validation.

The next speaker was Dr John Kadavil (US FDACDER-OTS-OSIS, Silver Spring, MD) [4] who spoke about the transition many laboratories are making to electronic notebooks (ELN) for bioanalytical assay development, validation and sample analysis documentation. A series of questions were posed by the speaker as considerations for ELN users including server storage location, archival and retrievability of records. One of the highlights was the importance of presenting an overview of the ELN workflow to the auditor for ease of navigation and inspection. Some other notable considerations included ensuring property security controls, maintaining the application in a controlled manner as well as understanding how well your ELN environment is integrated with other software. Lastly, Dr Kadavil informed ELN users that paper-based notebook factors apply to electronic data capture systems as well.

Next, David Scharberg from Pharmaceutical Outsource Solutions (Santa Clara, CA) gave a presentation on 'A third-party auditor's perspective on trends in regulated bioanalytical compliance and enforcement'. Mr Scharberg's gave a few recommendations regarding guidance and dissemination of 483 notices. He gave several examples of unnecessarily redacted $483 \mathrm{~s}$ that upon further review may have been able to provide more useful information to the audience.

The second session on Day 1 focused on partnerships between private and public institutions to accelerate medical research. The session was chaired by Dr Farhad Sayyarpour (Ricerca Biosciences, Painesville, $\mathrm{OH}$ ) and Anne-Francoise Aubry (Bristol-Myers Squibb, Princeton, NJ). Dr Johan Lutman, VP Neuroscience Clinical Development at Eisai (Woodcliff Lake, NJ), discussed the pros and cons of closed and open innovation. Dr Lutman stressed the nonprofit, precompetitive consortia model has been generally successful at advancing commons goals between academic and private organizations. However, this must be balanced against 'consortia fatigue' in which there are 
not enough resources to contribute to the expanding number of groups.

Dr Adrienne Tymiak, Executive Director of Bioanalytical and Discovery Analytical Sciences at BristolMyers Squibb (Princeton, NJ), also gave a presentation on the power of precompetitive cooperation with the IQ Consortium (International Consortium for Innovation and Quality in Pharmaceutical Development) and the Allotrope Foundation. The IQ Consortium is dedicated to augmenting the capability of member companies to develop transformational solutions that benefit patients, regulators and the broader research and development community. The Allotrope Foundation is collaborating to deliver an innovative framework for laboratory data based on open standards.

The next presentation was from Dr Amanda Paulovich from the Fred Hutchinson Cancer Research Center (Seattle, WA). Her presentation focused on efforts underway to create a community assay repository (CAR) for standardizing and harmonizing multiplex proteomic measurements. Fundraising is underway to support the development of 'off-the-shelf' multiple reaction monitoring assay kits for all human proteins.

Session 3 on day 1, titled 'The latest in large-molecule bioanalysis', was chaired by Dr Marie Rock (WIL Research, Ashland, OH) and Dr Stephanie Farmer (BioData Solutions, Eagleville, PA). The session began with Dr Chris Rusconi from Regado Biosciences (South San Francisco, CA) . Dr Rusconi gave a detailed presentation on their investigation and conclusions into the association of high level anti-PEG antibodies with first exposure severe allergic reactions to their anticoagulant aptamer, pegnivacogin.

Dr Daniel Sexton, Senior Director of Pharmacology and Preclinical Development at Dyax (Burlington, $\mathrm{MA}$ ) gave a presentation on drug tolerance challenges in enzyme-inhibitor-based neutralizing antibody assays. Dr Sexton presented data and approaches within two case studies. One of his conclusions was that an understanding of the structural and biochemical interactions leading to bioactivity can support the use of a binding assay for neutralizing antibody detection.

Lastly on Day 1 of the Regulated Bioanalytical Section, Dr Laura Salazar-Fontana, Global Strategic Advisor for Immunology and Biomarkers at Sanofi (Framingham, MA), detailed a risk-based approach to immunogenicity evaluation.

Following three sessions in the Regulated Bioanalysis Workshop on day 1 there were two sessions titled 'New modalities in biotherapeutics' and 'Microsampling other than DBS' on day 2.

Emerging biotherapeutics are becoming a larger proportion of the development portfolios in the pharmaceutical industry [5]. The session of new modalities in biotherapeutics chaired by Rick Steenwyk (Pfizer, Groton, CT) and Lori Payne (Intertek, San Diego, CA) was started with a presentation on the reassessment of immunogenicity risk based on clinical data. All pharmaceuticals produce therapeutic and unwanted effects, while immunogenicity is a concern when developing biotherapeutics. Initial immunogenicity assessment is often based upon nonclinical immunogenicity profile which is not generally predictive of clinical immunogenicity [6]. In his presentation, Eric Wakshull (Genentech, South San Francisco, CA) showed a case study of a structurally novel biotherapeutic (one-armed antibody) where the immunogenicity risk-based assessment was updated as data from Phase I and II clinical trials became available and was used to justify more scarce sampling in Phase III.

The following presentations focused on PK bioanalysis of new modalities including ADC payload and nanomedicines. Joseph Tweed (Pfizer, Groton, $\mathrm{CT})$ discussed the challenges presented in ADC payload bioanalysis within the regulated discipline and the lessons learned in the application of various sample preparation techniques, chromatography choices and mass spectrometric detections. For examples, triple quadrupole and HRMS were compared for the quantitative measurement of a certain ADC payload. Incomplete fragmentation of the sodium adduct using triple quadrupole impacted the assay selectivity and sensitivity; however, HRMS in the full scan mode allowed simultaneous detection of multiple ionized species and offered the option to add isotopic contributions, which resulted in an increase of the signal to noise ratio. Coincidentally, the Vendor Talk of this session by Mark Wrona (Waters Corporation, Milford, MA) showcased how advances in HRMS hardware and data processing were driving enhanced selectivity and the dramatic impact that the technology was having on both small and large molecule quantitation.

The third presentation by Susan Low discussed another type of targeted therapeutics, Accurin ${ }^{\mathrm{TM}}$, developed by BIND Therapeutics (Cambridge, MA) [7]. Accurin BIND-014 is a nanomedicine where the chemotherapy drug docetaxel is physically entrapped without chemical modification. Docetaxel release from the particle is diffusion based and is temperature dependent. Traditional docetaxel bioanalysis does not distinguish between encapsulated and released docetaxel. Novel methods for separation of these fractions and the FDA guidance on bioanalysis of nanoparticles [8] were discussed in the presentation.

Microsampling continues to be a topic of considerable interest. The main reason for this lies with ethical aspects - animal number reduction and refinement of sampling procedures according to the $3 \mathrm{R}$ principles 
(reduce, refine and replace). The final session of regulated workshop chaired by Fumin Li (PPD, Richmond, VA) and Yongjun Xue (Celgene, San Diego, CA) addressed the most current applications and directions of microsampling other than DBS in support of the discovery and regulated studies. Sheldon Leung (Pfizer) presented an approach to analyze microsampled whole blood on the Gyrolab platform for the ligand-binding assays at Pfizer [9]. The approach has been used for over 2 years to support monoclonal antibody projects in discovery.

The other two techniques presented in this session were volumetric absorptive microsampling (VAMS) by Thomas Menkee (GlaxoSmithKline, Upper Merion, PA) and capillary microsample (CMS) by Larry Mallis (Frontage Laboratories, Exton, PA). The VAMS technique absorbs a fixed volume of blood $(10 \mu \mathrm{l})$ within $4 \mathrm{~s}$ with low volume variation across wide hematocrit range, which makes it a promising alternative that shares the benefits of other microsampling techniques, yet can reproducibly collect precise samples independent of hematocrit and reduce sample preparation complexity [10]. However, the CMS technique uses a flexible plastic capillary tube lined with ethylenediaminetetraacetic acid (EDTA) to obtain approximately 15-20 $\mu$ l of plasma sample. Small-metered sections of the plasma section of the capillary are then cut into multiple sections and stored for the initial analysis, reassay, and incurred sample reanalysis (ISR). With this approach a complete PK curve can be determined from a single mouse thereby reducing the number of animals in each study [11].

At the end of this session, a plenary talk titled 'Challenges in regulatory review of bioanalytical data' by Chongwoo Yu (FDA, Silver Spring, MD) brought the focus back to regulatory guidance. Clinical pharmacology data is pivotal in ensuring the delivery of the right drug, in the right dose, at the right time, and it has significantly influenced the risk/benefit assessment and labeling recommendations [12]. Consequently, the reliability of the bioanalytical methods and data are of considerable importance and the solid footing in drug development. Nine case examples were presented to highlight the utility and importance of bioanalysis and the challenges that are often faced in conducting regulatory review of bioanalytical data.

\section{Discovery bioanalysis}

Day 2 began with plenary talk from Dr Jack Hoppin (inviCRO, Boston, MA) about development of instrumentation and software informatics for the imaging of radiolabeled compounds and their distribution. The first Discovery session followed the plenary session and was titled 'Adjusting our bioanalytical strategies to study innovative biotherapeutic modalities', chaired by Dieter Drexler (Bristol-Myers Squibb, Wallingford, CT) and Mary Wells. The central theme of this session focused on adaptability and adjusting bioanalytical strategies when studying biotherapeutics. First speaker, Dr Tim Olah (Bristol-Myers Squibb, Princeton, NJ), began the session with a perspective on early discovery of alternative modalities, points to consider (efficacy, toxicity, complexity), assay availability and validation. As biopharma shifts from small-molecule entities to increasingly larger molecules - peptides, proteins, antibodies and ADCs - bioanalytical research evolves in parallel.

Method development is an iterative process that continuously considers qualification of the assay, followed by refinement, quantification and confirmation. In the case of a disulfide-rich cyclic peptide, unit resolution mass spectrometers such as triple quadrupoles and ion traps were insufficient in sensitivity and inefficient fragmentation led to loss of signal intensity. A novel survivor-selected ion monitoring approach was developed to allow sensitive and specific detection of the target while all other contaminates underwent MS/MS fragmentation noise reduction [13]. Bioanalysts face many challenges - sample limitations, low concentrations and complexities of matrices - resulting in the everchanging role of the bioanalyst and evolution of bioanalysis paradigm.

The next two speakers discussed the bioanalysis of hybrid molecules. Dr Hongyan Li (Amgen, Thousand Oaks, CA) discussed adjusting LC-MS/ MS assays for different peptide-antibody therapeutics, additionally comparing and contrasting ligand binding versus LC-MS. As hybrid molecules are comprised of multiple components, critical assays are needed to evaluate PK properties (exposure and distribution) and stability of peptide and linker (potential biotransformations). While traditional ligand-binding assay strategy can be utilized to measure bound peptide and total antibody, it is limited by the requirement of multiple reagents and the need for two separate assays. An integrated LC-MS/MS assay was developed to monitor a surrogate peptide from the Ab's comlpementary determining region (CDR) while simultaneously monitoring the peptide warhead. This integrated LC-MS/MS assay did not require specific reagents and concurrently provided $\mathrm{PK}$ and assessment of in vivo biotransformation.

The application and utility of the integrated LC-MS/MS assay was captured in three case studies. The first study applied this assay to a peptide-mAb fusion protein with three different constructs to determine which had favorable $\mathrm{PK}$, in vivo stability, and molecular integrity. Case study 2 was an example of 
peptide- $\mathrm{mAb}$ conjugates. The integrated LC-MS/MS method allowed for faster $\mathrm{Ab}$ screening and faster linker screening of succindyl 4-(N-maleimidomethyl) cyclohexane-1-carboxylate (SMCC) noncleavable linkers and cleavable disulfide linkers. The last case study focused on a reagent-free approach using PEG protein precipitation, enabling differential protein precipitation based on protein size while removing excess albumin as compared with a traditional metha$\mathrm{nol} /$ acetonitrile [14] or isopropyl alcohol (IPA) protein precipitation [15]. PEG protein precipitation resulted in wide working dynamic range with low $\% \mathrm{CV}$ s in serum and plasma.

The session ended with Dr M Violet Lee (Genentech, South San Francisco, CA) giving examples of LC-MS/MS PK assays for ADCs of past, present and future ADC platforms. ADC complexity is due to inherent heterogeneity and in vivo biotransformations. ADC complexity propels the need to develop a suit of PK assays which analyze key components: total $\mathrm{Ab}$, unconjugated drug and conjugate, measured either as conjugated antibody (amount of $\mathrm{Ab}$ associated with drug) or ADC (acDrug, amount of drug still conjugated to the $\mathrm{Ab}$ ) [16-18].

ADCs can be conjugated with either cleavable (enzymatic or chemical) linkers or noncleavable linkers. Past PK assays were exemplified in the enzymatic cleavage version of the acDrug assay was implemented across multiple clinical and nonclinical studies. The present acDrug PK assay development and optimization challenges for disulfide-linked drugs that require chemical cleavage was more complex than enzymatic cleavage, requiring optimization of reaction buffers and reaction kinetics. Orthogonal LC-MS methods were implemented to monitor complete drug release as part of assay development. There was a propensity for conjugation sites to affect assay development due to the Ab's specific chemical [19] and structural properties around the conjugation site. The trends in susceptibility of assay drug release were consistent with in vivo stability. New assays require adaptability and flexibility as each ADC platform may require its own acDrug assay optimization due to potential effects of different conjugation sites. While acDrug PK assay platform can be modified and applied to ADCs with different cleavable linkers, the future platform will include noncleavable linkers to monitor either peptide-linker-drug or single amino acid linker drug in place of release drug.

\section{Biotransformation}

The biotransformation section of the APA held sessions on Monday (day 1) and Tuesday (day 2) as well as a joint session with the APA Discovery section on Wednesday (day 3). The biotransformation plenary talk was given Monday afternoon by Dr Ian Blair of University of Pennsylvania (Philadelphia, PA) and focused on his work in the area of biomarkers as it relates to mitochondrial dysfunction. Topics in the biotransformation sessions covered a wide range of scientific endeavors from the more biologically orientated, 'Applications of 3D models/in vivo models/3D models for metabolism', chaired by Cyrus Khojasteh (Genentech, South San Francisco, CA) and Mehran Moghaddam (Celgene, San Diego, CA) to a 'Stereochemisrty' session chaired by Greg Walker (Pfizer, Groton, CT) and Kirk Henne (Amgen, Thousand Oaks, $\mathrm{CA})$. During the $3 \mathrm{D}$ model session a panel discussion was held to discuss in vitro cell culture tools available for better assessing ADME and toxicology endpoints. The participants included Onyi Irrechukwu (Hepregen, Medford, MA), Susanna Roe (Insphero, Brunswick, ME), Albert Li (In vitro ADMET Laboratories, Malden, MA), Matthew Shipton (H HREL, North Brunswick, NJ). The take home messages were that the 3D hepatic cultures comprised of several cell types including hepatocytes co-culture with nonparenchymal cells. In liver, the nonparenchymal cells include stellate cells, sings ideal endothelial cells, Kupffer cells, biliary epithelial cells, and immune cells such as leukocyte so and lymphocytes. All these cell types participate in the normal function of liver in its response to drugs and toxins. Liver function is tightly dependent on the complex assembly of these cell types and their sinusidal assembly. Therefore, using only one type of liver cells, that is, hepatocytes, in studying metabolism and response to cellular damage does not provide a complete picture. Assembly of hepatocytes and some of the nonparenchyma like cells into a 3D structure restores their ability to function more normally with the caveat that no in vitro assay will ever fully represent the in vivo setting. The panel participants were all in agreement with pros and cons of the in vitro assays and limitations of 3D systems. Nevertheless, they all successfully introduced their systems and thoroughly discussed their utilities. For a better understanding of these products, we recommend visiting the websites of each of the aforementioned groups. We also believe that each research organization should decide on their own when to employ 3D cell cultures in their workflow in order to maximize their return on investment.

In addition to these topics separate sessions were also held, discussing: transporters and their various roles in drug-induced liver injury, nephrotoxicity and drug-drug interaction (DDI), a session on PK/PD receptor occupancy, a session on pharmacologically active metabolites and final session on protein damage and chemical toxicity. 


\section{Summary}

The 2015 Applied Pharmaceutical Analysis (APA) conference is a relevant and impactful meeting particularly to those in the disciplines of regulated bioanalysis, discovery bioanalysis and biotransformation. The scope of the APA conference continues to offer small- and large-molecule scientists the opportunity to stay current with the state of current regulatory trends within the industry and provides a collaborative environment to share new ideas and technology that enable our science. Additional information and updates regarding the upcoming APA conference can be found on the Boston Society's website [20].

\section{Acknowledgements}

The authors thank Elena Tufa (The Boston Society, Lexington, MA) for the coordination and planning of the 2015

\section{References}

1 US FDA-center for drug evaluation-office of translational sciences-office of study integrity and surveillance-division of generic drug bioequivalence evaluation? www.fda.gov/AboutFDA/CentersOffices/OfficeofMedical

2 USC 262. Regulation of biological products, from Title 42 - The Public Health and Welfare.

http://uscode.house.gov/view.xhtml?req

3 Database of CGMP facilities and bioequivalence sites. www.fda.gov/ForIndustry/

4 US FDA-center for drug evaluation-office of translational sciences-office of study integrity and surveillance. www.fda.gov/AboutFDA/CentersOffices

5 Kinch MS. An overview of FDA-approved biologics medicines. Drug Discov. Today 20 (4), 393-398 (2015).

6 Gorovits B, Wakshull E, Pillutla R et al. Recommendations for the characterization of immunogenicity response to multiple domain biotherapeutics. J. Immunol. Methods 408, 1-12 (2014).

7 Hrkach J, Von Hoff D, Ali MM et al. Preclinical development and clinical translation of a PSMAtargeted docetaxel nanoparticle with a differentiated pharmacological profile. Sci. Transl. Med. 4(128), 1-11 (2012).

8 US FDA. Guidance for Industry: Liposome Drug Products. 2015 .

www.fda.gov/downloads/Drugs/Guidance

9 Joyce AP, Wang M, Lawrence-Henderson R et al. One mouse, one pharmacokinetic profile: quantitative whole blood serial sampling for biotherapeutics. Pharm. Res. 31, 1823-1833 (2014).

10 Denniff P, Spooner N. Volumetric absorptive microsampling: a dried sample collection technique for quantitative bioanalysis. Anal. Chem. 86, 8489-8495 (2014).

11 Jonsson O, Palma Villar R, Nilsson LB et al. Capillary microsampling of $25 \mu \mathrm{L}$ blood for the determination of
APA Conference. In addition, the authors thank Montserrat Carrasco-Triguero (Genentech, South San Francisco, CA), Eric Woolf (Merck, West Point, PA), Johanna Mora (Bristol-Myers Squibb, Princeton, NJ) and other members of the 2015 APA Organizing Committee for their participation in the thorough review and editorial suggestions used in the preparation of this manuscript.

\section{Financial \& competing interests disclosure}

The authors have no relevant affiliations or financial involvement with any organization or entity with a financial interest in or financial conflict with the subject matter or materials discussed in the manuscript. This includes employment, consultancies, honoraria, stock ownership or options, expert testimony, grants or patents received or pending, or royalties.

No writing assistance was utilized in the production of this manuscript.

toxicokinetic parameters in regulatory studies in animals. Bioanalysis 4(6), 661-674 (2012).

12 Yu C, Bashaw ED. Regulatory perspective of bioanalysis from a clinical pharmacology reviewer standpoint: do you see what I see? Bioanalysis 6(4), 505-510 (2014).

13 Ciccimaro E, Ranasinghe A, D'Arienzo C et al. Strategy to improve the quantitative LC-MS analysis of molecular ions resistant to gas-phase collision induced dissociation: application to disulfide-rich cyclic peptides. Anal. Chem. 86, 11523-11527 (2014).

14 Ouyang Z, Furlong MT, Wu S et al. Pellet digestion: a simple and efficient sample preparation technique for LC-MS/MS quantification of large therapeutic proteins in plasma. Bioanalysis 4, 17-28 (2012).

15 Liu G, Zhao Y, Angeles A et al. A novel and cost effective method of removing excess albumin from plasma/serum samples and its impacts on LC-MS/MS bioanalysis of therapeutic proteins. Anal. Chem. 86, 8336-8343 (2014).

16 Kaur S, Xu K, Saad OM et al. Bioanalytical assay strategies for the development of antibody-drug conjugate biotherapeutics. Bioanalysis 5, 201-226 (2013).

17 Gorovits B, Alley SC, Bilic S et al. Bioanalysis of antibodydrug conjugates: American Association of Pharmaceutical Scientists Antibody-Drug Conjugate Working Group position paper. Bioanalysis 5, 997-1006 (2013).

18 Liu A, Kozhich A, Passmore D et al. Quantitative bioanalysis of antibody-conjugated payload in monkey plasma using a hybrid immuno-capture LC-MS/MS approach: assay development, validation, and a case study. J. Chromatogr. B 1002, 54-62 (2015).

19 Shen BQ, Xu K, Liu L et al. Conjugation site modulates the in vivo stability and therapeutic activity of antibody-drug conjugates. Nat. Biotechnol. 30 184-189 (2012).

20 The Boston Society. www.bsat.org 